\title{
TEE-aided Write Protection Against Privileged Data Tampering
}

\author{
Lianying Zhao and Mohammad Mannan \\ Concordia University, Montreal, Canada \\ \{z_lianyi,mmannan\}@ ciise.concordia.ca
}

\begin{abstract}
Unauthorized data alteration has been a longstanding threat since the emergence of malware. System and application software can be reinstalled and hardware can be replaced, but user data is priceless in many cases. Especially in recent years, ransomware has become high-impact due to its direct monetization model. State-of-the-art defenses are mostly based on known signature or behavior analysis, and more importantly, require an uncompromised OS kernel. However, malware with the highest software privileges has shown its obvious existence.
\end{abstract}

We propose to move from current detection/recovery based mechanisms to data loss prevention, where the focus is on armoring data instead of counteracting malware. Our solution, Inuksuk, relies on today's Trusted Execution Environments (TEEs), as available both on the CPU and storage device, to achieve programmable write protection. We back up a copy of user-selected files as write-protected at all times, and subsequent updates are written as new versions securely through TEE. We implement Inuksuk on Windows 7 and 10, and Linux (Ubuntu); our core design is OS and application agnostic, and incurs no run-time performance penalty for applications. File transfer disruption can be eliminated or alleviated through access modes and customizable update policies (e.g., interval, granularity). For Inuksuk's adoptability in modern OSes, we have also ported Flicker (EuroSys 2008), a defacto standard tool for in-OS privileged TEE management, to the latest 64-bit Windows.

\section{INTRODUCTION AND MOTIVATION}

The first known crypto-ransomware dates back to 1989 (only file/directory names were encrypted [39]; see also [64]). Crypto-based attack vectors were formally introduced by Young and Yung in 1996 [78] (see also [79]). After the CryptoLocker attack in 2013, robust crypto-ransomware families have been growing steadily, with a large number of attacks in 2016 (see the F-Secure ransomware "tube-map" [17]). Examples of recent high-impact ransomware attacks, include [38], [66], [21], [10], [2], [57], affecting individuals and enterprise/government systems alike. An IBM X-Factor survey of 600 business leaders and 1021 consumers in the US reveals the effectiveness of current ransomware attacks: $70 \%$ of affected businesses paid the ransom ( $46 \%$ of businesses reported to have been infected); individual users are less willing to pay (e.g., 39\% users without children may pay ransom for family photos vs. 55\% users with children). For

Network and Distributed Systems Security (NDSS) Symposium 2019 24-27 February 2019, San Diego, CA, USA

ISBN 1-891562-55-X

https://dx.doi.org/10.14722/ndss.2019.23197

www.ndss-symposium.org a conservative estimate of financial loss, a recent end-toend measurement [23] shows that over \$16 million USD in ransoms has been collected from 19,750 potential victims over a two-year period. Ransomware's direct monetization has benefited from pseudo-anonymous payment systems such as Paysafecard.com, prepaid/gift cards, and crypto currencies (e.g., Bitcoin), and not-easily-traced indirect payments such as sending SMSes to premium numbers [31].

Common anti-malware approaches relying on binary signatures are largely ineffective against ransomware (see e.g., [55]). Some solutions rely on system/user behavior signatures, exemplified by file system activity monitoring, e.g., [30], [55], [14], [29]. To complement detection based solutions (or assuming they may be bypassed), recovery-based mechanisms may also be deployed, e.g., Paybreak [32] stores (suspected) file encryption keys on-the-fly, right after generated but before encrypted with the ransomware's public key.

On the other hand, general (rootkit-level) malware that targets to corrupt/delete user data for various purposes also has long existed, e.g., wiper malware [71], without demanding a ransom, which is worse in terms of recovery. In addition to recent incidents (e.g., [19]), this is also exemplified by the notorious virus CIH [27] (1998), which erased files and even corrupted BIOS. Several countermeasures against generic rootkit attacks have also been proposed, focusing on intrusionresiliency and forensics (e.g., S4 [60]), and preventing persistent infection (e.g., RRD [12]). FlashGuard [24] is the only proposal focusing on rootkit ransomware, which leverages the out-of-place write feature of modern SSDs, providing an implicit backup. It requires modifying SSD firmware and a trusted clock within the SSD (currently unavailable). We discuss academic proposals in more detail in Section VII.

Inspired by traditional data access control (e.g., permissionbased and read-only protection in file systems ${ }^{1}$ ) and backup mechanisms, we shift the focus from detection/recovery to data loss prevention against rootkit malware (including ransomware). If user files could remain unmodifiable by malware even after the system compromise, no reactive defense would be necessary-enabling data loss prevention. However, this new paradigm requires that writes must be allowed at certain times when new data is added or changes are made. Of course, rootkit malware can also make malicious changes to the protected files once write access is enabled.

We propose to achieve data loss prevention with an appendonly history-preserving backup storage framework, where pro-

\footnotetext{
${ }^{1}$ Note: read-only folders/files enforced by the OS (e.g., Windows 10 "controlled folder access" [44]), only prevents unprivileged access.
} 
tected files are always exposed read-only to applications and the operating system, and append operations are allowed only in a secure manner. In our threat model with rootkit malware, such read-only access (equivalent to write protection) need to be enforced by hardware/firmware to counteract privileged software. Considering that there exists no commerciallyoff-the-shelf (COTS) storage device with this append-only feature, we resort to trusted execution environments (TEEs, see Section II) to allow programmable write protection. We use a host-side TEE to enforce the (append-only) logic and confidentiality-protect an authorization secret, and a deviceside TEE to allow write access only with that secret. In a broader sense, the placement of secrets and logic across multiple TEEs relies on the underlying assumptions and support from available hardware (refer to Section IV for further discussion).

By choosing the self-encrypting drive (SED, see Section II) as the device-side TEE, we design Inuksuk, ${ }^{2}$ to protect existing user files from being deleted or encrypted by malware. Inuksuk functions as a secure data vault: user-selected files are copied to a write-protected SED partition, and the secret to allow write-access is cryptographically sealed to the machine state (i.e., the genuine Inuksuk and the correct hardware platform), and hence, allowing file writes to the data vault only from the trusted environment. Deletion/modification outside the environment will fail due to the write protection. Meanwhile, access to the read/writable copy on the original partition is not affected (processed at the next commit). Inuksuk takes rootkit ransomware as a major threat but also works against any privileged unauthorized data alteration.

Inuksuk works without prompting for any user secret. It merely appends to existing data. Files created or modified on the original partition are all treated the same way, and copied (i.e., committed) onto the protected partition as new versions in the host-side TEE (referred to as TEE thereinafter if not otherwise specified), without overwriting existing files. However, user consent is needed for solicited file deletion, e.g., when the user no longer needs a document. Our assumption is that deletions are done occasionally and preferably in batch (disk space is relatively cheap). Inuksuk comes with a builtin mini file browser for the user to select and delete files in TEE. Files on the protected partition remain accessible as read-only, allowing the user to mount the drive elsewhere for recovery without TEE in the case of malware detection or system corruption (see Sections IV and VI).

We choose to instantiate the host-side TEE using Trusted Platform Module (TPM) chips, and CPUs with Intel TXT or AMD SVM (see Sections IV-C and IV-D for reasons, and Section II for background). Due to the exclusive nature (which is also a great security benefit) of the TXT/SVM environment, during file operations on the protected partition, the system is unavailable for regular use. We thus provide two access modes for both home and enterprise users: a) Network-based. User devices and computers are connected to a regular network storage system; a dedicated Inuksuk computer then copies user data from the regular storage to its protected storage, and thus user experience is not affected. b) Stand-alone. The user works with the Inuksuk-equipped device, e.g., a laptop. In this

\footnotetext{
${ }^{2}$ Inuksuk is an Inuit word with multiple meanings, including: a (food) storage point/marker.
}

mode, system unavailability is reduced with custom scheduling policies (e.g., triggered during idle periods, akin to Windows updates)

While Inuksuk can provide strong security guarantees, its implementation faces several technical challenges. For example, the TXT/SVM environment lacks run-time support and we must directly communicate with the SED device (for security) and parse the file system therein (involving performance considerations). Note that the use of Intel SGX is infeasible for Inuksuk, as SGX allows only ring-3 instructions, i.e., cannot access the disk without the underlying (untrusted) OS. Also, the user OS is unaware of the TXT/SVM sessions, so the devices (i.e., keyboard/display for secure user interface) are left in an unexpected state (see Section V). Note that there have been a series of attacks based on SMM (System Management Mode) over the past few years, some even affecting Intel TXT [75]. This does not pose a serious threat to Inuksuk, because of its particular setting, e.g., exclusiveness with no bootloader/hypervisor/OS involved (discussed more in Section VI). Last but not least, TXT's exclusiveness also protects it from many side-channel attacks that are highly effective against non-exclusive TEEs such as Intel SGX and ARM TrustZone.

\section{Contributions.}

1) We design and implement Inuksuk against root-privileged data tampering, in a radical shift in threat model from existing academic/industry solutions. We target loss prevention of existing data, instead of detection/prevention of malware/ransomware.

2) Inuksuk's design is tied to the combination of established and standardized trusted execution environments (in our prototype, SED disks and Intel TXT/AMD SVM with the TPM chip). Integrating TXT/SVM, TPM, and SED/Opal together in a seamless way with a regular OS (Windows/Linux) is non-trivial, but offers a significant leap in the arms-race against malware. Our solution, together with the ported Flicker (which will be both open-sourced), solves several engineering/performance problems when faced with exclusive TEEs (e.g., DMA with TEE, disk/file access, display), which can also be methodologically useful for other TEE applications.

3) We implement Inuksuk on both Windows and Linux (Ubuntu). The core design is OS-agnostic. Our prototype achieves decent disk access performance within the OS-less TXT/SVM environment (around 32MB/s read and 42MB/s write), when committing files to the protected partition. The regular disk access to original files from the user OS remains unaffected, i.e., all applications perform as before.

4) We also port the state-of-the-art in-OS trusted execution manager Flicker [42] to Windows 10 64-bit (Flicker's latest version only supported Windows 7 32-bit). This advances privileged trusted execution (as opposed to user-space only) up to date, available to other secure processing applications in modern 64-bit operating systems.

5) Beyond unwanted modifications of protected data, Inuksuk can be used as a generic secure storage with fine-grained access control, enabling read/write operations and data encryption (with Inuksuk-stored keys), if desired. Inuksuk in the stand-alone mode is locally enforced without any network dependency, and operates with a small TCB. 


\section{BACKGROUND}

In this section, we briefly explain certain terms and background information to facilitate understanding of the Inuksuk design and prototype implementation hereinafter.

Trusted Execution Environment (TEE). Modern CPUs usually support a special secure mode of execution, which ensures that only pre-configured unaltered code can be executed, with integrity, secrecy and attestability; and provides a form of isolation from both other software/firmware and physical tampering. TEEs can be exclusive, preempting and suspending other code (e.g., Intel TXT and AMD SVM), or concurrent, co-existing with other processes (e.g., Intel SGX, ARM TrustZone, and AMD SEV [46]). There are also privileged (TXT, SVM, SEV and TrustZone) TEEs and unprivileged TEEs (SGX). This is about whether privileged instructions (e.g., I/O) are allowed or untrusted OS has to be relied on to provide such services.

Technically, TEEs cannot function alone. For the purpose of storing measurements (to be matched with that of the code being loaded) and secure storage of execution secrets, a Secure Element (SE) is used in conjunction. It can be part of the processor die, an integrated chip, or a discrete module.

In this paper, we use the term TEE in a broader sense, i.e., referring to the aforementioned secure processing feature of any processor-equipped devices that operates with secrets, in addition to that of PC and mobile platforms. For instance, more and more IoT devices make use of microcontrollers with ARM TrustZone for Cortex-M such as Nordic nRF91 [72] and NuMicro M2351 [51]. Also, there are legacy devices with secure processors (cf. smart cards [13]).

Intel TXT and AMD SVM. Trust Execution Technology (TXT) is Intel's first "late launch" technique, aiming at establishing trusted execution any time after system reboot, without relying on what has already been loaded (e.g., BIOS). It is exclusive, removing software side-channel attack vectors and with the help of VT-d [7], largely defends against violations from the I/O space. AMD SVM (Secure Virtual Machine) is a similar technology, which we consider as equivalent to TXT, with slight differences, e.g., it does not involve an explicit ACM (Authorized Code Module, such as TXT's SINIT) and has fewer requirements for the measured program (called SLB in SVM and MLE in TXT). Nevertheless, they share the security properties we need for Inuksuk so we refer to them undistinguished as TXT/SVM hereinafter. When individual terms are used, the discussion is specific to one.

Note that TXT/SVM has different positioning than Intel SGX and can handle privileged instructions, e.g., device I/O as needed by Inuksuk. They do not replace each other. TXT is widely supported by many commercial-off-the-shelf Intel CPUs, ${ }^{3}$ and SVM (SKINIT) is available in almost all modern AMD CPUs.

TPM. Trusted Platform Module is a microchip, serving as the SE for TEEs (usually TXT or SVM). Its volatile secure storage includes PCRs (Platform Configuration Registers) where the run-time measurement can be stored and matched with. They can not be directly accessed but only extended (i.e., replaced

\footnotetext{
${ }^{3} 47$ CPUs released in 2018 as of Dec. 6 (ark.intel.com).
}

with the cryptographic hash value of its original value concatenated with the new measurement). Its non-volatile secure storage is called NVRAM, which is accessible in the form of index (a numeric identifier). NVRAM indices can be allocated and deallocated and there can be multiple of them.

Sealing. Short for cryptographic sealing, it is a special mode of encryption, provided by TEEs/SE, where the key is tied (in various ways) largely to the machine state, in the form of measurement. Measurement is the chaining of the loaded programs in sequence, e.g., concatenation of hashed values in the SE. Any single bit of change in loaded programs will cause a mismatch of measurement, making the reproduced key different, and thus render the decryption (unsealing) to fail. In this way, platform binding is achieved.

Flicker [42]. Before the introduction of Flicker, Intel TXT was mostly applied with the pilot project tboot, which deals with boot-time trusted execution (cf. OSLO [28] for AMD SVM). The ability to switch between the regular OS environment and the trusted execution was not available. Flicker enables such transitions, e.g., interrupting and saving states for the OS, initiating the TXT/SVM session, performing trusted operations and resuming the OS. The trusted operations are encapsulated in what is called a PAL (Piece of Application Logic) and thus OS-agnostic. It satisfies what is needed in Inuksuk.

Self-Encrypting Drive. With the same interface and form factor, regular hard drives or SSDs can be equipped with a built-in crypto engine and certain enhancement to the controller, thus providing on-device encryption and access control. Such devices are called Self-Encrypting Drives (SEDs) [8]. Instead of more generic secure processing, functionalities are mainly related to media access control and data encryption (e.g., with the so-called Device Encryption Key). Most SEDs offer fine-grained protection, such as dividing media space into ranges and splitting read/write accesses. In addition to the standard ATA interface, Trusted Computing Group (TCG) also has its open standard named Opal/Opal2 [69] for SEDs. What Inuksuk needs is the fine-grained programmable write protection enforcement (data secrecy not as a goal).

\section{THREAT MODEL AND ASSUMPTIONS}

1) We assume that malware/ransomware can acquire the highest software privileges on a system (e.g., root/admin or even ring-0 on $\mathrm{x} 86$ ), through any traditional mechanisms (often used by rootkits), including: known but unpatched vulnerabilities, zero-day vulnerabilities, and social-engineering. Root-level access allows malware to control devices (e.g., keyboard, network interface), GUI, installation/removal of device drivers.

2) Before deployment of Inuksuk, the user system is not infected by any malware. We primarily protect preexisting data at the time of malware/ransomware infection, and provide best-effort protection thereafter for later added/updated files until the malware/ransomware is detected (or a ransom is demanded).

3) We do not detect/stop the execution of malware, or identify its actions. Instead, we protect integrity of user data on a protected partition and ensure data accessibility. If the OS is completely corrupted or inoperable, the user can install 
a new OS copy or boot from another media (e.g., USB) to access her data.

4) In the specific case of ransomware, we deal with the most common variants (i.e., cryptoviral extortion), and exclude those that simply lock access to system resources without using encryption (non-encrypting ransomware [52]) or deletion, and those that threaten to publish information stolen from the user (doxware or leakware [50]).

5) We assume all hardware (e.g., the CPU/chipset and the storage device), microcode/firmware and other architecture-shipped modules (e.g., TXT's SINIT, see Section II) are properly implemented by the manufactures, and the user is motivated to choose a system with no known flaws. An example of such a flaw is a series of recently identified implementation bugs [43] in SED firmware implementations that highly affect data secrecy (refer to Section VI for details).

6) Attacks requiring physical access are excluded (e.g., no evil-maid attacks). We only consider a computer system potentially infected by malware/ransomware from the network or a removable drive.

7) We assume that after infection, ransomware will act immediately; i.e., it will find target user files, encrypt them, and then demand a ransom without much delay (e.g., few minutes/hours, cf. [24] vs. months). If the attacker waits, he risks of losing control, e.g., through an OS/antimalware update. With every patched computer, the attacker loses money, and thus cannot remain hidden for long. To accumulate file updates, the attacker may wait for some time (i.e., long enough to collect sufficient content that the user may care), before asking for the ransom. We term such attacks as persistent ransomware, and discuss them more in Section VI, item (d).

\section{DESIGN}

We first define our design goals for Inuksuk, then explore available/possible choices with one or multiple TEEs placed at different locations of the storage data flow and their implications; we illustrate our generalized design and its workflow by choosing readily available COTS options. We explain certain technical challenges/choices in Section V. The discussion will start with and be based on the stand-alone mode of Inuksuk. The network-based mode, which follows, just shifts the standalone complex of TXT/SVM, TPM and SED to a network location. The terms ransomware and malware in our setting of unauthorized data alteration can be interchangeably used.

\section{A. Design goals}

We list our goals, and briefly sketch the key ideas to fulfill such goals in Inuksuk.

a) Enforcement by device. Rootkit malware must not be able to modify or delete protected files. We place them in a write-protected mode (read-only) all the time in the user OS. The write protection must not be bypassed or broken by rootkit and thus it must be enforced by the storage device, where the protected partition resides, instead of any software on the host. Therefore, without the appropriate authentication key (a highentropy random value, e.g., 256-bit long), the partition cannot be unlocked, even if the OS is compromised (malware gains all software permissions). b) TEE-aided write protection. To allow updates, lifting the write protection is inevitable at a certain point in time, during which protected files can be compromised by rootkit malware. In Inuksuk, write operations to the protected partition only occur inside a TEE. The authentication key is protected by, and bound to, this TEE (inaccessible from outside). All changes are treated as new versions (retaining historical versions) and infrequent (batch) deletions are performed with user interaction or through policies.

c) Minimal application interference. Applications (including the user OS) should operate as is. As the original files are untouched by Inuksuk and accessed the same way by applications, normal application I/O is not hindered (even for direct $\mathrm{I} / \mathrm{O}$ as in disk utilities). File copies on the protected partition are available as read-only, which should not concern regular applications.

d) Minimal user involvement. User experience should not be significantly affected. A normal user experience is preserved in Inuksuk with the separation of the original and protected copies. To reduce system unavailability for the stand-alone mode, the update/commit process should be scheduled during idle hours, and all updates to the original copy are cached to be committed as a new version periodically (e.g., every 8-12 hours). The user can also choose the network-based mode as discussed in Section IV-F to avoid unavailability. The user is involved only when files must be deleted (sometimes including removal of old versions), and manually triggering Inuksuk (for immediate commitment of cached files, when the important files are just edited/added).

Non-goals. Inuksuk is designed to act more like a data vault than a traditional backup system; e.g., we commit user data a few times a day in batches, instead of syncing updates instantly. Also, it adds another layer of protection to high-value user data, complementing existing backup systems. Namely, OS/application binaries should not reside on the protected partitions; regular user data may already be backed up (e.g., to certain cloud storage). We provide robust data integrity against advanced attacks at the expense of losing some data due to ransomware attacks (e.g., user updates to a file during the commit period). Also, data confidentiality is currently a non-goal (to facilitate unhindered operations of common applications); i.e., the ransomware can read all protected user data, and read/modify the OS/unprotected partitions. However, confidentiality and controlled read access can be easily supported; e.g., encrypting data under Inuksuk-protected keys, and enabling password-based access control for read operations on selected files.

\section{B. Trusted file versioning}

We treat all write operations to the original copy (consolidated in one commit) as adding new files to the protected partition (automatically approved, similar to S4 [60]), which poses no threat to existing files, leaving only solicited file deletion (as opposed to version deletion) with user intervention. Any committed update to an existing file creates a new version, instead of overwriting the current version (the latest one being under the original file name) so that historical changes committed are all retained on the protected partition. For space management, we leave it to the user to either clean 
up in the mini file browser we developed (see Section V-E for details), or configure an auto-deletion policy based on aging (e.g., after 1-2 years) or version-limiting (e.g., maximum 100 versions). The eventual choice is largely determined by the user's budget and needs. Our simple versioning may not impose a significant burden on the storage space, considering: a) We commit changes to the protected partition through scheduled invocation of Inuksuk; users can explicitly trigger the updater to commit important changes immediately, which we believe would be infrequent. So the number of versions that will be stored for a continuously updated file would still be limited, e.g., 1-4 times a day. Auto-save in applications or file access-time change do not trigger an update (it is only on the original copy). b) Nowadays, disk storage is less costly and user computers are usually over-provisioned. To improve storage utilization, specifically for large files, more spaceefficient versioning algorithms may be adopted (e.g., S4 [60]). Also, refer to the file selection principles discussed below for optimal scenarios.

User consent is mandatory when files (as opposed to versions) are to be deleted. File versions can optionally be deleted manually by the user. We allow deletion operations in the mini file browser within the trusted environment, where the user is asked to select explicitly which file(s) to delete. User consent is not needed in the case of auto-deletion of versions. Direct file deletion in the protected partition outside the trusted environment will be ignored;deletion of the original copy in the unprotected partition will not be synchronized to the protected partition. We also hide old versions from the user OS to help usability. When a new version is committed, we rename the previous copy by appending its timestamp with the file name, and keep the new version with the original name.

Automatic stale version deletion. To relieve users from deleting unnecessary old versions of the same file, Inuksuk can be configured to automatically delete such versions after a certain time (aging) or number of versions (version-limiting). The retention duration should be long enough to hurt ransomware's business model. For example, if an attacker needs to wait more than a year to monetize his ransomware, it might become much less attractive than now. Defenders are likely to generate reliable detection mechanisms (e.g., signatures) within the wait period, and even be able to identify the attackers. Calculation of the time duration (for aging) must be done appropriately, if there is no trusted time source available within the TEE (e.g., TXT/SVM). As rootkits can change system time, file creation/update time as available from the user OS file system cannot be trusted. A simple solution could be to use digitally signed time value from an NTP service, ${ }^{4}$ where the signature verification is done within the TEE. The signed value can be obtained through the user OS, and must be sent for each file commit session. The trusted updater must store the last accepted signed value along with NTP verification keys, and check the new timestamp to detect replay (the time value should always be increasing).

File selection principles. Although technically we are not restricted in terms of file types, to optimize the user's budget and needs, the user is recommended to follow a few principles:

\footnotetext{
${ }^{4}$ See Section 6.2.2 at http://www.ntp.org/ntpfaq/NTP-s-config-adv.htm. Alternatively, time-stamping services, implemented by several CAs (following RFC 3161), can also be used.
}

- Targeted. The user identifies what she cannot afford to lose (must pay ransom for). For example, an HD movie of $5 \mathrm{~GB}$ that can always be redownloaded should not be selected for protection. However, we do consider such valid needs (e.g., of a movie editor), where corresponding high-capacity disk space is assumed affordable.

- Minimal. The user should reduce redundancy from the selected files. For instance, a mobile app developer may have (multiple copies of) the Android source tree (tens of GBs) which she compiles a couple of times a day. The files changed by compilation are not necessarily those she cannot afford to reproduce. Therefore, she may just choose source files reflecting her work.

- Prioritized. For instance, the update frequency for an important project report (e.g., 1-3MB) should be set higher in the policy than the user's favorite songs (e.g., MP3s, 300MB).

\section{TEE placements}

Based on our design goals, Inuksuk can be hypothetically constructed around the following entities:

1) Host. The processor-centric system where software (including user applications) runs and where data is generated/consumed. Benign and malicious operations cannot be naturally distinguished.

2) Disk. The storage device where legitimate user data (from user applications) is supposed to be securely stored. No additional mechanism other than physical safety is assumed.

3) TEE. There might be multiple TEEs. They can be equipped on either the Host or the Disk, or both. The TEE has the properties and purposes discussed in Section II. In light of the Disk's constraints, we further model all TEEs for three types of functionalities: I) Policies. This applies to general-purpose TEEs (as with that of the Host), where the user/vendor can provision arbitrary storage rules, e.g., write protection. II) Authentication. We consider the most constrained application-specific TEEs on the Disk to be able to authenticate commands from the Host with a configured secret, e.g., a TLS certificate and/or a password. III) Secure protocol. A secure communication protocol between the Host and the Disk may be supported (in addition to authentication), which ensures integrity and confidentiality, e.g., the untrusted OS cannot learn what is being sent and if it tries to manipulate the data it will be detected.

4) Interface. This is how the Disk is connected to the Host physically, as exemplified by SATA, USB and Ethernet. We consider both secure and insecure interfaces. ${ }^{5}$ In a threat model with no physical access, direct links with endpoints inside the TEE can be treated as secure. Otherwise, for example, Ethernet (WAN, where other nodes are involved) or SATA relying on untrusted kernel drivers is susceptible to interception. A secure interface cannot provide message authenticity if it is not dedicated to the TEE. For instance, outside the TEE (for concurrent TEEs) or when the TEE is not active (for exclusive TEEs), untrusted software on the Host can still send anything to

\footnotetext{
${ }^{5} \mathrm{~A}$ secure interface ensures data integrity and confidentiality (but not necessarily authenticity) in the discussion hereinafter.
} 
the Disk, unless there is a way to block access (e.g., with the TrustZone protection controller [10]) to the Interface from untrusted software at all times.

The storage data flow goes between the Host and the Disk through the Interface. The TEE's location can shift along the data flow across different entities. In addition to the autonomous enforcement of predefined policies (e.g., appendonly), certain administrative access to the Disk is also needed for space management, i.e., there must be a convenient way for the user to delete files or change the configuration. In the following, we consider a few possible constructions with different TEE placements. We use the subscript to indicate where the TEE resides, e.g., TEE $E_{\text {Disk }}$ means a TEE is on the Disk.

$\mathbf{T E E}_{\text {Disk }}$ alone. If $T E E_{\text {Disk }}$ enforces the append-only and history-preserving logic on its own, $T E E_{\text {Host }}$ is not necessary. Figure 1-a depicts this construction. However, it lacks a trusted user interface for the administrative access. This might be solved by adding 1) a physical switch, to allow occasional full access, or 2) a secondary dedicated interface, assuming there exists a trusted host for performing administrative tasks. Therefore, we do not consider this construction for our prototype.

TEE $_{\text {Host }}+$ secure Interface. In contrast to $T E E_{\text {Disk }}$ alone, if there is only $T E E_{H o s t}$ enforcing the logic, regardless of whether the Interface is secure or not, our goals still cannot be achieved. For insecure Interface, malicious enitity can directly intercept traffic and corrupt data. For secure Interface, which might seem sufficient for channel integrity and secrecy, untrusted software on the Host can also do harm at the data origin. When $T E E_{H o s t}$ is not in control, other privileged code can access the secure Interface and modify/delete the stored data. The root cause is that the Disk has no TEE to authenticate $T E E_{\text {Host }}$.

TEE $_{\text {Host }}+$ dedicated secure Interface. On top of the construction above, if $T E E_{\text {Host }}$ comes with an I/O partitioning mechanism (e.g., the TrustZone protection controller), the secure interface can be configured in a way that when an exclusive $T E E_{\text {Host }}$ is not active or outside a concurrent $T E E_{\text {Host }}$, no software can access it (Figure 1-b). As an advantage, this allows any storage device to be used as the Disk. Also, with $T E E_{\text {Host }}$, the administrative access can be provided to the user. This construction may become feasible in the future when ARM-based desktop/laptop computers can efficiently run modern desktop OSes [45].

$\mathbf{T E E}_{\text {Host }}+\mathbf{T E E}_{\text {Disk }}+$ insecure Interface. As $T E E_{\text {Disk }}$ is usually not general-purpose (i.e., supporting arbitrary code to be provisioned), we need $T E E_{H o s t}$ to be used in conjunction to achieve our purpose. A minimum support from such $T E E_{\text {Disk }}$ is the use of certain secret for encryption or access control (e.g., SED and Kinetic storage), which can serve as the basis for write protection (read-only). If $T E E_{D i s k}$ supports a secure communication protocol, e.g., TLS, a secure Interface is unnecessary. In this case, as long as the shared secret (or its public-key equivalent) is properly handled in $T E E_{H o s t}$, a secure channel is formed, equivalent to a secure Interface. $T E E_{\text {Host }}$ enforces all the logic. Note that unprivileged TEEs (see Section II) can be used here, since the requirement for a secure Interface has been relaxed. See Figure 1-c for an illustration. This corresponds to the construction of Pesos [33], as detailed in Section VII. This construction is also missing a trusted

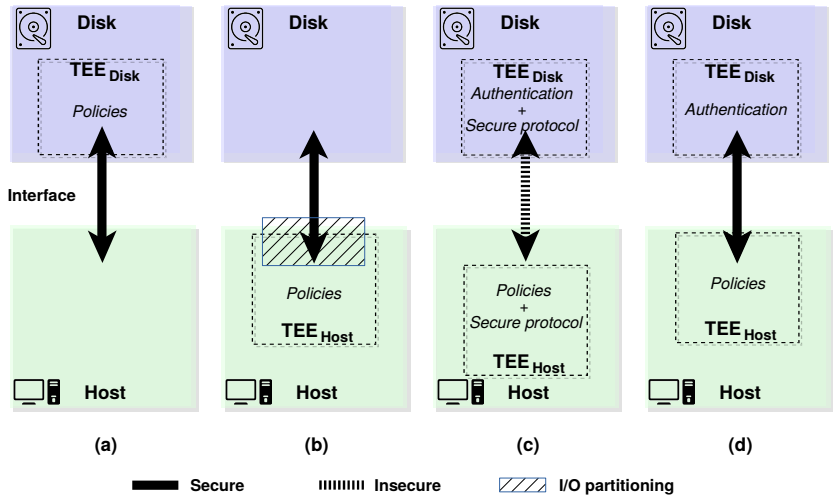

Fig. 1: Example constructions with varying TEE placements. An Interface arrow not terminating inside $T E E_{\text {Host }}$ means the TEE is unprivileged and relies on other software (e.g., the OS) to perform I/O. Privileged TEEs (with the arrow inside) only own Interface when active, hence the need for certain I/O partitioning (e.g., TZPC) to block it to other software when the TEE is not active, making Interface dedicated

UI for administrative access (forged input can be entered in $T E E_{\text {Host }}$ thus deleting unintended files), as unprivileged TEE only interacts with the user through the untrusted OS.

$\mathbf{T E E}_{\text {Host }}+$ TEE $_{\text {Disk }}+$ secure Interface. Peripherals directly connected to a PC with exclusive/privileged $T E E_{H o s t}$ is considered to have secure Interface. In this case, $T E E_{D i s k}$ that does not support a secure communication protocol is applicable (e.g., Opal SED). $T E E_{\text {Disk }}$ authenticates $T E E_{\text {Host }}$ in cleartext; this could be problematic if attackers gain physical access or $T E E_{\text {Host }}$ is non-exclusive/unprivileged. Privileged $T E E_{\text {Host }}$ provides a trusted UI for administrative access (forged UI outside $T E E_{\text {Host }}$ cannot be authenticated by $T E E_{\text {Disk }}$ without the secret). We choose this construction to implement Inuksuk; see Figure 1-d.

\section{Design choices}

According to the discussion above, we expand on further considerations and requirements on the chosen construction of $T E E_{\text {Host }}+T E E_{\text {Disk }}+$ secure Interface. Note that the design choices are only based on available COTS devices for this prototype. The high-level design is generalizable to other $T E E_{\text {Host }}$ and $T E E_{\text {Disk }}$ (as shown in Section IV-C).

Host-side TEE. To satisfy secure Interface, $T E E_{\text {Host }}$ must be privileged (Figure 1-d); otherwise, the OS may manipulate the I/O traffic. Also, our observation shows that if a TEE is privileged, it is usually exclusive (i.e., not allowing other software to run in parallel) unless there is hardware I/O partitioning as with ARM TZPC, to avoid resource access conflict or contention. Actually, as an advantage with privileged TEEs, no other applications (including the OS) can have the chance to even observe what is running inside, as anything else is discarded/suspended, avoiding software side-channel attacks.

We use Intel TXT/AMD SVM as the host-side TEE for Inuksuk. Therefore, our discussion hereinafter will refer to $T E E_{\text {Host }}$ as TXT/SVM. The more recent Intel SGX does not run privileged code for $\mathrm{I} / \mathrm{O}$ access. ${ }^{6}$

\footnotetext{
${ }^{6} \mathrm{SGX}$ has also faced several side-channel attacks due to its concurrent nature, e.g., Foreshadow [11], Branch Shadowing [36], and cache attacks [20].
} 
Programmable write protection. As the ideal $T E E_{D i s k}$ (a general-purpose on-disk TEE, Figure 1-a) is unavailable (as of writing), and it also lacks a proper trusted UI on the Host for administrative access, we resort to storage devices with an application-specific TEE. $T E E_{\text {Disk }}$ needs to be able to communicate with $T E E_{\text {Host }}$ for the write protection (programmability). We expose write access to the protected partition only inside $T E E_{\text {Host }}$. Some off-the-shelf secure USB drives offer write protection [4]. However, it is either in the form of a physical switch/button to be pressed by the user, or a key pad on the USB device itself, where a password can be typed (like a closed TEE inaccessible from outside).

The self-encrypting drive (SED, see Section II) satisfies programmability, with one or more secrets for authentication or media encryption. Also, SED has the advantage of supporting fine-grained protection ranges with separate $\mathrm{read} /$ write permissions, which is important as we constantly allow read access, and deny write access from the user OS. Fine protection granularity also allows the protected partition to coexist with the unprotected OS and other files in the same drive, instead of requiring a dedicated disk. The only disadvantage, as far as Inuksuk is concerned, is that SED does not support secure communication protocols thus requiring a secure Interface. The legacy ATA Security password can also be considered device-enforced write protection (without media encryption). However, it is a non-solution for Inuksuk, because only one-way locked-to-unlocked transition is allowed (SEC4:SEC5 [62, p. 13]), i.e., relocking requires power reset, whereas Inuksuk needs the ability to switch back and forth.

Minimal TCB. Although a full-fledged OS in TXT/SVM (e.g., tboot with Ubuntu) can be used to perform trusted operations, it is preferable to keep a minimal trusted computing base (TCB), for both auditability (e.g., avoiding numerous complex components) and maintainability (e.g., avoiding measuring large and varying files). Moreover, it is technically more involving, because the trusted operations occur in the midst of an active user OS execution (considering the time/effort needed to save and restore various states for both OSes). Therefore, we develop our own logic as a small-footprint, native program in TXT/SVM with no external dependencies.

Separation of the protected partition from the original. Technically, we can write file updates immediately on the protected partition. However, unsolicited/frequent write attempts, such as updates from the automatic save feature in text editors (i.e., not initiated by the user clicking on the "Save" button), will create too many versions on the protected partition and make the system unusable due to frequent switch between regular and trusted environments; note that, TXT/SVM is exclusive, and writing file updates may also take noticeable time. Therefore, we leave user-selected files where they are, and make a copy onto the protected partition on the SED. All subsequent updates happen to the original files without write protection. The user can then decide when to commit changes to the protected partition (no versioning on the original partition), manually, or automatically at certain intervals (e.g., every 8-12 hours).

File-system in TXT/SVM. For protected write operations, we cannot simply pass the raw sector information (sector number, offset, number of bytes and the buffer) to TXT/SVM as we perform file-based operations, and the user also must select files (not sectors) for deletion. Therefore, the TXT/SVM program must be equipped with a file system.

Data mobility and recovery. The SED can also contain an unprotected partition where the OS resides, because of the fine granularity of protection ranges, while sometimes users may treat it as a stand-alone data drive. In either case, when data recovery is needed (e.g., the OS is corrupted or compromised), the user can simply reboot from different media on the same machine or mount the SED on a different machine. The data will be readily accessible as read-only, hence aiding data mobility, thanks to the separation of read and write accesses. In case the user needs to update the files, a rescue USB, where all intact Inuksuk binaries are stored as well as a portable OS can be used to boot the same computer (where Inuksuk was provisioned). After booting with the rescue drive, the user can invoke the same updater in TXT/SVM for regular file access or deprovisioning (to remove the write protection).

\section{E. System components and workflow}

Refer to Figure 2 for an overview of our design. The system consists of the following components at a higher level (further technical details are discussed in Section V):

- Trusted updater. This is the core component of Inuksuk, and runs inside TXT/SVM. It is responsible for copying files from the original partition to the protected partition (in SED write access mode) as new versions, file listing (in a mini file browser), and showing file meta data to the user. Files in the original partition are selected to commit based on their last-modified timestamp.

- TPM. In conjunction with TXT/SVM, TPM makes sure that the secret (the SED password) is securely stored in its NVRAM storage, and can be unsealed only if the unmodified trusted updater is executed (as measured in TPM's platform configuration registers).

- Secure drive. An SED drive hosts the protected partition. Without the high-entropy key/password, its protection (i.e., write protection in our case) cannot be bypassed. Note that even with physical access to the drive, reinitializing the drive with the PSID (physical secure ID) printed on it will have all data lost.

- OS drivers. A few OS-dependent modules are needed to bridge the user, OS and the trusted updater, such as preparing the TXT/SVM environment. These modules do not have to be trusted after initial deployment, as the worst case is a DoS attack; see also Section VI, item (b).

Update policies. The update policies reflect user preferences and control how the trusted updater behaves (as primitive text files in the prototype for now), e.g., the age threshold, version limit, maximum file size, scheduled interval, etc. Moreover, the user can optionally configure certain checks to be enforced in TEE for the purpose of anomaly detection, e.g., ransomware maliciously triggered 100 versions of a protected file. At each run, a report is generated securely in TEE, e.g., showing a list of updated files.

The policies are sealed on the protected partition (can only be unsealed inside TXT/SVM). A plaintext copy is left on the unprotected partition for certain operations (e.g., scheduled 


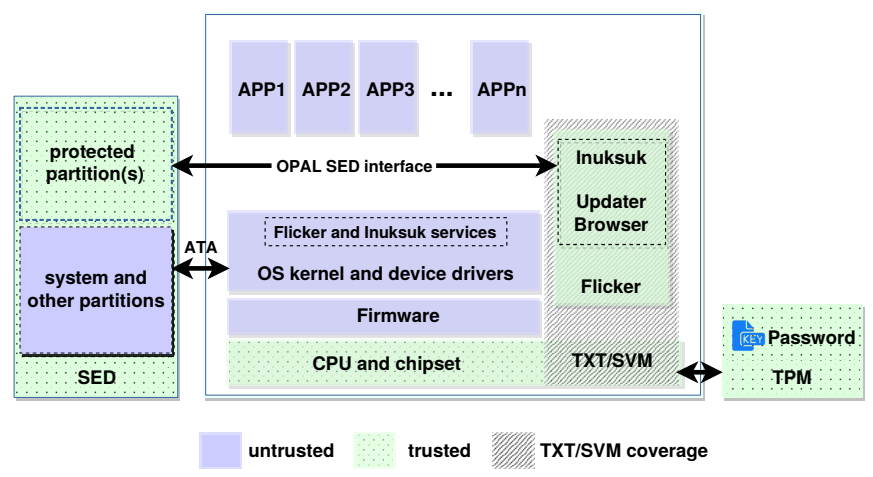

Fig. 2: System overview

updates based on an interval). Once in the trusted updater, the unsealed copy of policy can be used to verify the action's correctness (e.g., manipulated interval outside).

Workflow. The generalized workflow of Inuksuk is as follows: (a) At deployment time, a high-entropy secret is generated as the SED password and sealed into TPM (can only be unsealed in the genuine trusted updater). (b) The protected partition is created with the SED write protection. The user also selects the files to be protected, which are then copied to the protected partition in the first invocation of the updater. After the first-time copying, the user still interacts directly with her files on the original partition. (c) In everyday use, the protected partition is never touched (except for read-only access). As with certain cloud storage services, we use an icon on the original files to indicate which ones are under the protection of Inuksuk. (d) If the user adds or updates files on the original partition and is ready to commit her changes, she triggers the trusted updater, and without involving her to verify, changes are committed as new files/versions on the protected partition. The updater is triggered either manually, or automatically, e.g., via scheduled tasks, when the updating-application is closed, or when the system is restarting or shutting down. (e) When the user wants to delete files or old file-versions, she can manually trigger the updater to open a mini file browser, and make the selections.

\section{F. Network-based data vault}

The functionality of Inuksuk does not rely on any third parties (except the device manufacturer), as the trust is anchored in hardware/firmware and all its components are local. Although our explanation of Inuksuk is based on its stand-alone mode, there is no fundamental barrier in the design for it to be deployed as a remote/networked data vault. To provide users with a centralized network-based mode, as well as extending for enterprise and cloud storage services, we briefly explore a variant of Inuksuk where the key components, i.e., TXT/SVM CPU, TPM and SED, are shifted to a network location, forming a remote service. Users' data will remain protected at a central, Inuksuk-backed storage service, and users can keep using any device of their choice (i.e., with or without TEE, mobile or desktop, at home or in an enterprise). We believe that this variant can be used to protect security-sensitive/user files stored in cloud storage services like Dropbox and OneDrive, or enterprise storage services. Although such services are possibly backed by robust backup measures and strict security policies/tools, if infected, consequences can be high.
The construction goes as follows: any desktop/laptop/mobile device serves as the front-end directly used by the user. Through an account, the front-end is connected to a storage back-end, which plays the role of the "original partition" in our stand-alone setup, caching file updates. Eventually, an Inuksuk-equipped backup server, which has the TXT/SVMcapable CPU and chipset, as well as the SED (or more likely, an SED array), is connected with the storage back-end. The Inuksuk-server will periodically copy new/updated files from the storage server, and become unavailable during this period, which should not affect functionality, assuming the Inuksukserver is not used for other purposes. The storage back-end and user devices remain available all the time.

Once deployed correctly, without the high-entropy key sealed in TPM, no remote attacker can turn off the write protection and update/delete the protected files. Our threat model now assumes that the remote attacker can infect the storage and Inuksuk servers, in addition to user devices. As before, only the uncommitted files remain vulnerable, and after written to the Inuksuk protected storage, user files become safe against any data modification attacks. Content on the Inuksuk-equipped server can be maintained by enterprise IT administrators (e.g., for deleting old versions). The whole process is transparent to end-users/employees, and the files that need ransomware protection can be identified by enterprise policies. In the case of home users, the storage back-end and the Inuksuk-equipped server can physically reside on the same NAS (Network Attached Storage) device, assuming certain disruptions are tolerable.

\section{IMPLEMENTATION AND EVALUATION}

We have implemented Inuksuk for Windows 7 non-PAE, Windows 10 64-bit and Ubuntu 12.04. In this section, we discuss important technical considerations and choices, as well as performance evaluation/issues during the prototype implementation. Our techniques can also be useful for other OS-less I/O intensive TXT/SVM applications. We perform our development and testing on an Intel Core i7-2600@3.40GHz (and AMD FX-8350 8-Core @4.0 GHz), 16GB RAM, and Seagate ST500LT025 SED disk. The performance numbers are from the Intel machine. Also a few other computers are used for debugging and cross-validation. As an estimate of the TCB size, in addition to Flicker's codebase (2012-0618, v0.7), Inuksuk adds 5190 LoC (in the PAL, which runs in TXT/SVM, OS drivers excluded). Among this, fat_io_lib contributed 1852 (for FAT32), followed by TCG Opal (1507) and USB (1467). We used the tool LocMetrics [37] to count the lines with some trivial manual effort. There are also additional engineering-oriented technical details omitted here for brevity, such as resource handsoff with the OS for secure user interface (keyboard and display).

\section{A. Porting and using Flicker for TXT/SVM sessions}

Since Inuksuk's secure file operations occur alongside the user OS, a mechanism is required for jumping back and forth between the trusted updater and the user OS. It can be implemented as a device driver (in the user OS) dealing with parameters, saving the current OS state, processing TXT/SVM logic, and restoring the saved OS state when returned from the trusted updater. Several such operations are already handled in 
Flicker [42] (also refer to Section II), which we use as the base of our prototype.

Flicker supports only 32-bit non-PAE Linux and Windows 7 OSes (no update since 2012), which is a limitation for our prototype with modern 64-bit systems. We thus port Flicker to the latest Windows 10 64-bit, which may benefit the community for other in-OS secure processing with device I/O, or when the exclusive processing environment of TXT/SVM is desired (as opposed to other TEEs such as SGX and TrustZone). Similar to the 32-bit Flicker, our adaptation, especially for context saving/restoring, is also mostly based on heuristics, as the available Intel/AMD documentation does not specify what is preserved or affected by TXT/SVM. Here, we omit the technical challenges for this porting, for example, the 4level unity page table covering both physical and virtual, 32bit and 64-bit addresses; 64-bit context saving/restoring; the discontinued inline assembly, etc. Note that this process is far more complex than adapting user-space applications or kernel drivers/modules for 64-bit.

\section{B. Opal access to SED inside TXT}

All software outside TXT/SVM, including the OS and all its device drivers, is untrusted in Inuksuk. However, inside the TXT/SVM environment, there is no run-time device support, i.e., devices including any SED drive cannot be accessed by default. Therefore, we must implement standalone (and preferably lightweight, to limit the TCB size) custom driver for accessing SED devices inside TXT/SVM. Various SED protocols rely eventually on the SATA interface (ATA Command Set [65]), with two options to choose from: 1) ATA Security password [62] (e.g., prompted in BIOS at bootup). In this option, SEDs only differ with regular hard drives in that user data is encrypted on the media. 2) Dedicated security protocols (e.g., Seagate DriveTrust [56], IEEE 1667 [25] and Microsoft eDrive (all based on TCG Opal/Opal2 [67]). They implement support for multiple roles/users corresponding to multiple ranges, with separate passwords for write/read access.

Granularity in both protection ranges, and separate read/write permissions is important in our design. The same drive can host both protected and unprotected partitions (which cannot be achieved in Option 1). Thus Option 2 is more suited for our needs, and we choose to use TCG Opal to communicate with SED, as it is an open standard and widely supported by most devices. Actually, with TCG Opal we can define multiple ranges (corresponding to partitions) protected by different secrets. Then these secrets can be sealed with different programs (e.g., the updater is one) thus reducing the risk of exposing all protected data altogether as in a single point of failure. We leave this as future work. Note that Opal is merely the payload security protocol (SFSC) of the carrying interface, be it SATA or NVMe; NVMe supports two variants of TCG Opal, Opalite and Pyrite [68]. We anticipate that supporting NVMe (for higher performance) may require only trivial changes.

A few open-sourced tools can manipulate SED devices with OS support (in addition to proprietary tools for vendor-specific protocols); we have tested msed [53] (now merged into DTA sedutil [15]) and topaz-alpha [3]. They mainly rely on the I/O support from the OS, e.g., SCSI Generic I/O, in the ATA passthrough mode. However, our TXT/SVM PAL is OS-less with no run-time support. We decide to port functions from topaz-alpha [3] as needed. The porting process faces several engineering challenges, which we omit here for brevity.

\section{File system efficiency}

We handle updates to the protected partition at file-level instead of raw sectors (see Section IV). This requires at least basic file system functionalities implemented within TXT/SVM. To avoid rework, we tested several libraries, including fat_io_lib [1], ThinFAT32 [61], fedit [18], efsl [80], etc. FAT32 projects that are tightly coupled with external dependencies are excluded (e.g., the mainstream FAT32 support with Linux VFS inodes). But none satisfies both the two necessary features: 1) Buffering support. Usually, FAT32 access is sectorwise, while DMA requests need to handle as many sectors as possible to reduce per-request overhead. PIO access is not affected but it is by nature slow. Note that hardcoded prefetching for reads is an overkill (reading data never needed), and hardcoded write buffer will hang (waiting for enough number of sectors). 2) Multi-cluster support for space allocation. At the file creation time, and when a file grows in size, FAT32 must traverse all clusters to find free clusters to be appended to the cluster chain of the file. Interestingly, with all FAT32 projects we tested, only one cluster is allowed to be added (we do not see any performance problem for allowing multiple). Therefore, for a 50MB file taking 6400 clusters (8KB clustersize) and the partition having 131072 free clusters (1GB), it takes more than 800 million iterations.

We choose fat_io_lib for adaptation, because of its good buffering performance. To add multi-cluster support, for each iteration, we start with the cluster where we left off, instead of the first cluster of the partition. We emphasize that Inuksuk is not dependent on any specific file system, and thus FAT32 can be replaced with a more efficient one.

\section{Discussion on DMA inside TXT}

The necessity for Direct Memory Access (DMA) is ubiquitous, even for a light-weight program like the Inuksuk updater. For instance, USB keyboards are the defacto norm. Unlike other simpler protocols, the controller (e.g., EHCI [70]) requires several host-allocated buffers in the main memory (DMA chunks) for basic communication with the host (e.g., the periodic frame list). The controller accesses the buffers without the CPU's intervention, hence, direct memory access. Also, for data transfer to/from the hard drive (e.g., SED), The theoretical speed of ATA PIO modes [6] is very low. Taking into account the file system overhead, it needs 3-4 minutes to write a $100 \mathrm{MB}$ file, which is unacceptable from the user-experience perspective. In modern systems, DMA is usually enabled for disk access.

However, the fundamental protection of TXT (like all other TEEs) must prevent autonomous access from peripherals for the protected regions. The MLE (Measured Launch Environment, specific to Intel TXT) memory is included in either the DMA Protected Range (DPR) or Protected Memory Regions (PMRs), which is mandatory (cf. [26]). Consequently, since we cannot (and do not want to) exclude the MLE from DMA protection, we have to allocate the DMA chunks outside. In our specific case where physical attacks are excluded and no other 
$\gg \gg \gg \gg$ Mini File Browser v0.1 $<<<<<$

SPACE to toggle selection;

DEL to delete; ENTER to view metadata

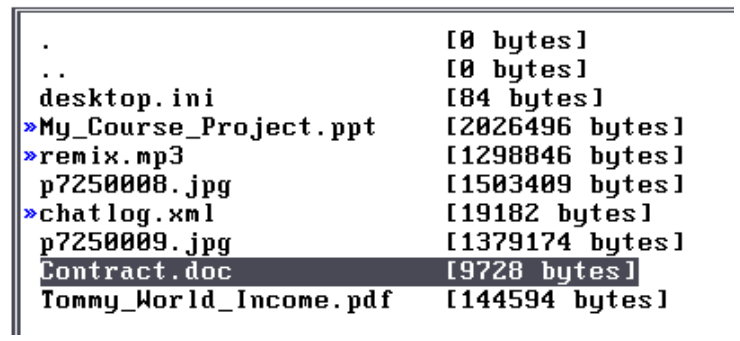

Fig. 3: A screenshot of the mini file browser inside the trusted updater. Selected files are designated with “»”; group selection can be specified by the first and last files.

code is running in parallel, exposing DMA regions outside the MLE does not pose a threat (more in Section VI, item (e)).

In this way, we implement our custom DMA support for both the USB keyboard and ATA DMA controller for data transfer. We also support PS/2 keyboard. Usually, DMA relies on interrupts, i.e., when the transfer is done, the interrupt handler will be notified to proceed to the next request (e.g., to maximize CPU time utilization in a multitasking environment). In our case, Flicker is not supposed to work with an interrupt-enabled workload (technically possible with some complex adaptation), and we merely need the performance boost through DMA, i.e., no multi-tasking and thus, requiring no interrupt support. Therefore, our custom DMA support works with polling. With DMA enabled, file transfer in the Inuksuk updater is 50-100 times faster than using just PIO (see Section V-E).

\section{E. Measuring disruptions}

Inuksuk does not introduce run-time performance overhead for user applications. However, when updates are being committed to the protected partition (file copying), the computer will be unavailable for regular tasks, due to TXT/SVM's exclusiveness. Such disruption is determined by both the file I/O performance and various factors, e.g., the file count/size. It mainly comes from data transfer for new versions, i.e., created/modified files. Deletion involves only flagging the files as deleted in the file system, and thus is quick. We have developed a light-weight file browser inside the trusted updater that allows the user to choose multiple files for deletion; see Fig. 3. With more engineering effort, graphical interface can also be created. There is no technical limitation for creating custom UI within TXT/SVM. Also recall that by configuring Inuksuk in the network-based mode, such disruption can be mitigated. The discussion here only focuses on the stand-alone mode.

File I/O evaluation. The file transfer speed determines the duration of disruptions, and affects user experience. However, we argue that the way we implemented DMA and our choice of the FAT32 library (as well as our adaptation to it) are confined by the engineering effort and time. Therefore, the numbers we show here should be just the lower bounds.

As micro-benchmarking, we executed 10 measurements on the files we selected with fixed sizes; see Table I. They are all one-way access, write-new/write-existing/read respectively.

\begin{tabular}{l|l|c|c|r|r|c}
\multicolumn{2}{l|}{} & Write/Existing & Write/New & \multicolumn{1}{c}{ Read } & OS & Penalty \\
\hline \multirow{2}{*}{$50 \mathrm{MB}$ file } & Mean & 43.93 & 41.69 & 32.17 & 68.77 & $53 \%$ \\
& SD & 3.40 & 0.31 & 0.09 & & \\
\hline \multirow{2}{*}{$500 \mathrm{~KB}$ file } & Mean & 26.46 & 8.09 & 16.67 & 14.29 & $43 \%$ \\
& SD & 1.18 & 0.43 & 5.26 & & \\
\hline
\end{tabular}

TABLE I: File transfer performance $(\mathrm{MB} / \mathrm{s})$ in the trusted updater from 10 measurements. For small files (e.g., 500KB), other overhead predominates the transfer time.

$50 \mathrm{MB}$ represents common media files and 500KB represents miscellaneous files of trivial sizes. Note that without our adaptation to enable multi-cluster allocation support, the creation of a $50 \mathrm{MB}$ file can be done only between $0.5-1 \mathrm{MB} / \mathrm{s}$, while overwriting of an existing file of the same size runs at about $40 \mathrm{MB} / \mathrm{s}$. To have a rough comparison with regular file transfer performance, we also list the corresponding speed in Windows aside (a 10-time average of reads and writes). The overhead percentage is calculated with the lowest speed of Inuksuk divided by the corresponding Windows value.

To demonstrate Inuksuk's performance in a realistic usage scenario, we invoke the trusted updater to copy 50 random photos (JPG files, size ranging from $1009 \mathrm{~KB}$ to $2416 \mathrm{~KB}$, totaling $85.6 \mathrm{MB}$ ) from the original partition to the protected partition. We measured the duration for 10 times, and the performance seems reasonable (mean: 23.3853 seconds), and relatively stable (standard deviation: 0.58989 ). This is a combination of read, write, file opening/closing, accumulating space fragments, etc. We also evaluated only the transition time between the OS and trusted updater. It varies between 2-4 seconds, including screen mode switching.

If we take into account any extra processing during file transfer, the time needed may also be affected. The basic versioning Inuksuk uses is not incremental, i.e., the whole of the source file on the original partition is copied over to the protected partition as a new version. We may consider some open-source version control systems like SVN/Git (or even the simple diff command) for incremental versions to save disk space. However, in that case, each time new files/updates are committed, the updater must scan the whole of both files for differences and then perform the transfer. Moreover, deletion is supposed to be very quick with non-incremental versioning (just flagging the file); with diff-like versioning, for each file the updater has to reassemble from all previous versions to form the latest one to be kept. The overhead could be significant in our setting (considering batch-deletion of versions). Also, for common file types such as images, videos, and rich documents (e.g., PDF, Word), incremental versioning may not save much disk space.

\section{SECURITY ANALYSIS}

In this section, we list various potential attack vectors, and discuss how they are addressed, or why they do not pose a threat (see Section III for our assumptions).

Since we shift the defense from detection/recovery to data loss prevention, we avoid common attacks such as whether new malware/ransomware can evade detection, whether it does privilege escalation, and how the encryption keys are generated. There are two basic questions in evaluating Inuksuk's effectiveness: 1) Outside the trusted environment, can malware/ransomware update files on the protected partition? No, without the high-entropy key sealed in TPM, software on 
the host system cannot break the write protection enforced by SED. 2) Inside the trusted environment (updater), can the malware/ransomware trick the user or the updater to write arbitrary content? The updater does not synchronize any file deletion from the original partition but only adds files from it. With the updater's integrity ensured by TXT/SVM, user I/O cannot be influenced by any external software.

(a) Forged user interface. Due to human users' inability to authenticate machines (cf. Stark [48]), rootkit malware may mimic the appearance of the intended application, where the user may leak secrets. However, the adversary will not benefit from it, as there is no UI in Inuksuk for prompting for the SED unlock secret (in fact, the unlock secret is unknown to users). Also, for manual deletion, there is no way to specify which files to delete from outside the trusted updater (files are selected in TXT/SVM right before they are deleted). In the end, without the genuine updater in TXT/SVM, the adversary cannot manipulate any file on the protected partition.

In addition to leaking secrets, forged UI can also help malware hide termination (skipping execution of Inuksuk), making the user believe the update has occurred (actually it was a fake one). To discern, the user can configure a perdeployment secret such as an avatar or short phrase to be shown in the trusted updater (mini file browser). Because of the exclusiveness and I/O isolation of TXT/SVM, rootkit malware will not learn this secret and thus is unable to forge a genuinelooking UI with the correct secret.

(b) Malicious termination, modification or removal of Inuksuk. A simple but effective attack against Inuksuk is terminating its kernel driver in the OS, or even completely removing it. Similar to rootkit malware's termination of hostbased anti-malware defenses, rootkit ransomware can easily launch this attack against Inuksuk. ${ }^{7}$ The pre-existing files on the protected partition remain immune to this attack; however, newly created or updated files thereafter are not protected. As with the forged UI attack mitigation, the user-defined avatar or short phrase can indicate the correct execution of Inuksuk in TXT/SVM, regardless of other tampering outside. Minimal user diligence is needed to just make sure the Inuksuk UI is regularly (automatically or manually) seen with this avatar or phrase. Note that modifying the Inuksuk updater's binary, which may reside on the unprotected partition, does not help the attacker; the SED unlock secret can only be accessed by the genuine Inuksuk updater (TPM unsealing).

(c) Known attacks against SEDs. Müller et al. [47] show that SEDs are also vulnerable to known attacks against software FDEs (e.g., cold boot, warm boot, DMA, and evil-maid). They also found a simple attack called hot plug, enabled by the fact that SEDs are always in a binary state of locked or unlocked. Once it becomes unlocked in a legitimate manner (e.g., usersupplied unlock passwords), the adversary can connect the disk to another attacker-controlled machine without cutting power, and can get access to protected data. In addition to these attacks, an adversary may also capture the cleartext SED secret/password from the SATA interface, e.g., by tapping the connection pins with a logic analyzers. Since all such attacks require physical access, i.e., desoldering a microchip, manipulating the connector or evil maid attacks, they are not viable

\footnotetext{
${ }^{7}$ Malicious termination can be made difficult by registering Inuksuk as a Windows Early Launch Antimalware (ELAM) driver.
}

for a scalable ransomware attack. More recently, different from the design limitations above, certain implementation flaws have been identified by Meijer and van Gastel [43], which severely affects SED security. To our understanding, the current SEDspecific flaws, e.g., user password and DEK not linked, mostly concern data confidentiality (with physical access), whereas Inuksuk's goal is data integrity (i.e., write protection). However, there exist undocumented vendor-specific commands $(\mathrm{VSCs})^{8}$ on certain storage devices that allow flashing unsigned firmware (directly or indirectly), which completely breaks $T E E_{\text {Disk }}$. Among SED disks, Crucial (Micron) MX100 and MX200 are vulnerable with such VSCs as reported by Meijer and van Gastel. We may have to choose those SED drives without such VSCs (e.g., after static analysis of firmware extracted via JTAG). Caution is always necessary when taking devices with firmware as part of the TCB (cf. the threat model of FlashGuard [24]). Last but not least, usually major SED manufacturers apply certain form of authentication/verification of the firmware before being updated to the drive, e.g., Secure Downloads and Diagnostics. ${ }^{9}$

(d) Attacks on TXT/TPM. Although TPMs offer some physical tamper-resistance, TPMs and similar security chips have been successfully attacked in the past (e.g., [28], [63], [58], [34], [73]); see also Nemec et al. [49]. However, with physical access excluded, we do not need to consider these attacks; also note that tapping TPM pins and DMA attacks require a malicious device to be connected. Regarding known softwareonly attacks against TXT, most such attacks are ad-hoc (e.g., the SINIT module flaw [76]), or version-specific; Intel has purportedly patched them in the subsequent versions, or at least the user is motivated to choose one that has no known flaws.

There are also attacks against TXT (e.g., [75]) that exploit the System Management Mode (SMM), an intrinsic part of the Intel X86 architecture, referred to as Ring -2. If the SMI (SMM interrupt) handler is compromised and SMI is left enabled, it can preempt the TXT execution and intercept trusted operations. Although no OS, hypervisor or bootloader runs in parallel with Inuksuk to trigger SMI (e.g., by writing to port 0xB2), certain micro-architectural behavior may facilitate such attacks, e.g., the CPU temperature sensor. Nevertheless, certain Intel CPUs leave SMI disabled after SINIT in TXT [26]; we can also explicitly disable SMI generation with the Southbridge (model-specific) upon entry to our code, as SMI is not needed in Inuksuk. This at least significantly reduces the attack time window to just the number of CPU cycles needed to disable it. Especially, for AMD CPUs [9], external SMI interrupts that assert after the start of SKINIT execution will be held pending (to ensure atomicity of SKINIT) until software subsequently sets GIF to 1 (internal SMIs are lost). We do not set GIF back to 1, and rely on polling instead (we do not gain extra performance with interrupts in our single-threaded environment). Another possible (powerful) attack avenue similar to SMM is vulnerable Intel Management Engine firmware [16]. Unless there is a pressing need for $\mathrm{ME}$, we suggest to disable it in a rigorous manner (for efforts and difficulties, see [54], as there is no architecture support for disabling ME and SMI elegantly).

\footnotetext{
${ }^{8}$ These VSCs are like regular commands sent through the SATA or NVMe interface, which can be done by any privileged code.

${ }^{9}$ Seagate (our SED) prevents counterfeit firmware: https://www.seagate. com/ca/en/solutions/security/
} 
(e) Compromise-then-DMA attacks. Although very unlikely to occur, we still consider a special situation where a DMA attack can be mounted but argue that it does not pose any threat. The remote adversary or malware can compromise a programmable peripheral (e.g., a functionality-rich gaming keyboard) and use it as the attack device. Then Inuksuk's exposed DMA buffer (not covered by Intel VT-d or AMD DEV protections) might be manipulated by that compromised peripheral (malware/ransomware). Those buffers only contain content to be written, while LBA location/sector count is still sent via regular I/O (WRITE-DMA-EXT), i.e., the adversary cannot point to the location of existing protected files. Therefore, encrypting the DMA buffers buys the adversary no more than doing the same outside Inuksuk, i.e., contaminating files on the unprotected partition. We can also reconfigure (every time inside TXT/SVM) DMA remapping in a way that the concerned range is only accessible to a specific PCI device (SED).

(f) Delayed attacks after deletion. Persistent ransomware can stay hidden for a long period (ranging from weeks to months), during which it just transparently decrypts encrypted data when accessed [22]. This can trick the user to believe that her data is intact (when viewed from within the OS). At some point, if she removes older versions to save space or auto-deletion is triggered, then the ransom can be demanded (i.e., no more showing the decrypted version).

The root cause of this problem is that OS-based file viewers (e.g., Microsoft Word), run outside the trusted environment and can be manipulated by rootkit ransomware arbitrarily, such as performing decryption before displaying a file to the user, or simply feeding a cached, unencrypted copy of the file. A straightforward countermeasure is to perform verification inside the updater before removing previous versions, e.g., by porting advanced file viewing tools in TXT/SVM, which can require significant effort. Such delayed attacks can be classified into two cases: 1) Ransomware-triggered file updates to exhaust version limits/space. 2) Updates piggybacking on legitimate user edits. Regular user files are either less frequently edited, or configured with a proper schedule to consolidate frequent edits. Therefore, the latter would take a long time for enough number of versions or age. We thus argue that the former would be more effective for the adversary. Nevertheless, Inuksuk can show an update log (with the list of files committed) to the user in the trusted updater on each run. It will raise an alert if the user notices files being committed that she has not touched. According to our file selection principles (see Section IV-B), the number of files being committed each time should account for a very small portion of all the protected files (e.g., 5 files out of 1000) unless the total number is also small.

In general, if auto-deletion with aging is enabled, we suggest the duration should be long, e.g., a year or two, depending on the size of the protected partition. Note that, delayed attacks risk being discovered and mitigated by anti-malware vendors, and thus we do not consider them a serious threat.

(g) Attacking auto-deletion with aging. If older file versions are automatically deleted after a preset threshold (e.g., 365 days), a straightforward threat is clock source manipulation. Rootkit ransomware can adjust the system time (to a far future date) to fool Inuksuk to believe the versions are already too old to be kept. To address this, Inuksuk can be configured to only trust a signed NTP time from a remote server, absence of which will stop auto-deletion (see Section IV-B).

\section{RELATED WORK}

There are many solutions dealing with user-level malware/ransomware for data protection; only FlashGuard [24] targets rootkit-level ransomware. However, some solutions against data manipulation by rootkit malware (not specific to rootkit-level ransomware) are close to Inuksuk in spirit. We discuss several examples from each category.

Reverse-engineered keys. Early-day ransomware had the (symmetric) file encryption keys embedded in their obfuscated binaries, or stored in a C\&C server. Keys could be recovered by reverse-engineering their code or intercepting $\mathrm{C} \& \mathrm{C}$ traffic. Ransomware now generally uses a public key to encrypt a random file encryption key, and the private key remains only at the attacker's machine (cf. [78]), and thus much more resilient than before; however, implementation flaws [74] may still be leveraged to recover encryption keys. An exemplary umbrella solution is NoMoreRansom [5], clustering file recovery efforts from several public and industry partners. However, relying on ransomware authors' mistakes is a non-solution, and finding such exploits may be too late for early victims.

Offline and online backups. An obvious recovery-based countermeasure against malware/ransomware is to make offline backup of important data regularly (on media disconnected from the computer or with device-enforced write protection, as ransomware also attempts to erase accessible backups). Although simple in theory, effective deployment/use of backup tools could be non-trivial, e.g., determining frequency of backups, checking integrity of backups regularly (see Laszka et al. [35] for an economic analysis of paying ransom vs. backup strategies). More problematically, the disconnected/write-protected media must be connected/unlocked (online) during backup, at which point, malware/ransomware can encrypt/delete the files (see [24], [41]). For cloud-based backup systems, such as Dropbox (centralized) and Syncthing.net (P2P), a potential issue is the size of their TCB (includes a full OS with multiple network-facing servers), which may lead to large-scale data loss, if compromised.

Rootkit-level solutions. S4 [60] is proposed as a self-securing storage entity behind a security perimeter, which records all file operations (like journaling or auditing) and retains old versions of user files. It is implemented as a network service (similar to NFS), and assumed to be resistant to compromise by a remote party (due to S4's limited outward interface). The usage scenario is focused on intrusion survival and forensics collection, in the case of an admin account compromise in a client machine. As S4 promptly stores all changes made to the client machine, as soon as possible, its storage overhead can be significant. To address this challenge, S4 makes use of novel compression and differential versioning techniques, which can benefit Inuksuk as well. Also, without any TEE to ensure execution integrity and secrecy, it involves the whole server infrastructure as the TCB, exposing many attack vectors. More likely than a full system compromise, if the admin account of S4 (or any similar backup system) is hacked, large volumes of data may be lost at once.

FlashGuard [24] proposes to modify the garbage collection mechanism of SSD firmware (assuming vendor support), so that for suspicious overwrites (i.e., first read and then written in a quick succession), a copy of the original data block is kept for 
a preset amount of time (e.g., 20 days). FlashGuard leverages a unique out-of-place write feature of modern SSDs (in contrast to regular hard drives), which provides an implicit backup of recently overwritten data blocks. The user is expected to detect any attack before the preset time elapses and perform the recovery from a separate machine; otherwise the data will be lost. The detection of suspicious overwrites can be an issue; e.g., ransomware can read and encrypt the file, and at some later point (i.e., not immediately to avoid being flagged), delete the file. However, this can be solved by retaining all deleted data blocks, at the expense of increased storage overhead. FlashGuard authors also do not specify the clock source to measure the preset time; SSDs do not offer any trusted clock, and relying on OS/BIOS could be fatal.

Rootkit-resistant disks (RRD [12]) are designed to resist rootkit infection of system binaries, which are labelled at installation time, and write operations to protected binaries are mediated by the disk controller. System binaries are updated by booting into a safe state in the presence of a security token. While effective against rootkit infection, RRD is infeasible against ransomware that targets regular user files (adding/updating will require reboot). Inuksuk's goals are complementary to RRD's and exclude protecting system binaries.

User-level solutions. Defenses are usually implemented as system services, kernel drivers (unprivileged adversary), or even user-land applications. For instance, Redemption [30] explicitly mentions that their TCB includes the display module, OS kernel, and underlying software. Redemption claims to provide real-time ransomware protection, by inspecting system-wide $\mathrm{I} / \mathrm{O}$ request patterns. Its detection approach involves a comprehensive list of features, with both content-based (entropy, overwriting and deletion) and behavior-based (e.g., directory traversal). In the end, a malice score is calculated to facilitate decisions. Redemption creates a protected area, called reflected file, which caches the write requests during inspection; the file is periodically flushed to disk (if no anomaly is identified). This ensures data consistency in case of false positives, i.e., if the suspicious operations is confirmed by the user to be benign, there is still the chance to restore the discarded data.

In an effort to achieve better universality and robustness, some proposals are purely data-centric (i.e., agnostic to program execution, checking just the outcome). E.g., CryptoDrop [55] focuses on file transformation information for individual files, regardless of where those transformations come from. It also claims to achieve early detection. It employs three novel indicators to detect suspicious file operations. Low file similarity before and after may indicate encryption but legitimate operations can also cause it (e.g., a blurred JPG file). Shannon entropy can be used in detecting encryption although compression also leads to high entropy. Last, file type changes (through content parsing) might not be robust enough with format-preserving encryption [59].

Although most ransomware mitigation techniques aim to detect/prevent ransomware as the primary goal, very few also focus on recovery, e.g., PayBreak [32]. Symmetric keys used by ransomware to encrypt user data are captured through crypto function hooking before they are encrypted with the adversary's public key, and then stored in a secure key vault. When infection is detected or a ransom is demanded, the user can retrieve the keys for decryption without paying the ransom.
PayBreak's crypto function hooking works for both statically and dynamically linked binaries, but only if the ransomware uses known third-party crypto libraries. Also, it is subject to evasion by obfuscation for statically linked ransomware. The key vault, even though encrypted with the user's public key and protected by the admin privilege, can still be easily erased by rootkit ransomware.

ShieldFS [14] is a copy-on-write shadowing filesystem reactive to ransomware detection, which is also based on I/O requests (I/O Request Packets - IRPs). Its methodology fits in the intersection of recovery-based solutions and data loss prevention, and thus is similar to Inuksuk in positioning. The detection portion also makes use of numerous behavioral features reflected from the IRPs. Specifically, ShieldFS's cryptographic primitives detection, different from PayBreak's, does not rely on hooking known crypto libraries, but captures inevitable properties of crypto primitives, such as the key schedule pre-computation of block ciphers. To achieve the claimed self-healing, on the first write attempt, ShieldFS keeps a copy of the original file in a protected location (only from userland processes); once an anomaly is detected, the changes made can be reverted with this copy, or otherwise it can be deleted at any time.

Microsoft BitLocker [77] is a widely-used (enterprise) data protection tool integrated with the Windows OS. BitLocker provides strong confidentiality guarantees through TPM-bound encryption. However, when a BitLocker-protected partition is unlocked after a successful boot (i.e., accessible to the OS and applications), there is no way to distinguish a malicious write attempt from legitimate ones, and thus making the protected data vulnerable to even user-level ransomware attacks.

For advanced data protection in iOS, Apple's secure enclave co-processor (SEP [40]) is also a form of hardware security feature, enabling memory encryption and credentials management (among other functions). The SEP communicates with the application processors (APs) via a mechanism called Secure Mailbox. From the limited public documentation, it appears that per-application access control is possible with SEP, therefore, decryption (and thus updates) can be only exposed to the right application.

Closest to Inuksuk in design components is Pesos by Krahn et al. [33] but with different goals and threat model; they use Intel SGX as $T E E_{\text {Host }}$ and Kinetic Open Storage as $T E E_{\text {Disk }}$. The high-level similarity is that $T E E_{\text {Host }}$, enforcing certain storage protection rules, connects to and is authenticated by $T E E_{\text {Disk }}$, and the Disk is capable of executing operations assigned by $T E E_{H o s t}$. The major contribution is the flattened abstraction layers and a rich set of storage policies exposed. Pesos assumes that only remote servers (the Host) are potentially malicious and the client machine is trusted, hence excluding (rootkit) ransomware/malware on the client machine. Therefore, trusted UI is no longer a problem, so the user can perform administrative operations (if needed) from the client, such as specifying which files to delete through encrypted network to $T E E_{\text {Host }}$. Since the kinetic storage supports secure communication (TLS), WAN network as an insecure Interface can be used. In addition, if placed in Inuksuk's setting, it suffers from the same issue of untrusted data source, i.e., there is no way to distinguish malicious writes from benign ones, unless all applications can be ported and contained in SGX or TXT/SVM. 


\section{CONCLUSIONS}

In summary, we propose to focus on data loss prevention, in an effort to address rootkit-level data alteration as exemplified by ransomware, a significant threat that remains largely unaddressed in current state-of-the-art solutions. We leverage the trusted execution environments (TEEs) available with modern computing devices and reason along various TEE placements between the host and the storage device. Intel TXT and AMD SVM in conjunction with TCG Opal SED are chosen for Inuksuk as our current prototype. Inuksuk leaves original user files in use with applications and exposes the protected copies as read-only all the time, and silently accepts creation/modification of the files by preserving previous versions. Users are only involved in file deletion occasionally in the trusted environment (e.g., for regular file deletion or in case the protected partition becomes full). Although our current prototypes are less than ideal (e.g., file transfer performance), we believe Inuksuk is a solid step towards countering rootkit ransomware. The source code of our prototypes will be made available through: https://madiba.encs.concordia.ca/software.html.

\section{ACKNOWLEDGMENT}

We are grateful to our shepherd Jonathan M. McCune for guiding us in the final version of this paper. We also thank the anonymous NDSS2019 reviewers for their insightful suggestions and comments. We also appreciate the help we received from the members of Concordia's Madiba Security Research Group. The second author is supported in part by an NSERC Discovery Grant.

\section{REFERENCES}

[1] "FAT16/32 file system library," http://ultra-embedded.com/releases/fat_ io_lib.zip.

[2] "The history of Cryptowall: a large scale cryptographic ransomware threat," https://www.cryptowalltracker.org/.

[3] "Library and utilities for manipulating TCG Opal compliant selfencrypting hard drives," https://github.com/tparys/topaz-alpha.

[4] "List of USB flash drives with hardware write protection," https://www.fencepost.net/2010/03/usb-flash-drives-with-hardwarewrite-protection/.

[5] "The no more ransom project," https://www.nomoreransom.org/en/ index.html.

[6] "Programmed I/O (PIO) modes," http://www.pcguide.com/ref/hdd/if/ ide/modes_PIO.htm.

[7] D. Abramson, J. Jackson, S. Muthrasanallur, G. Neiger, G. Regnier, R. Sankaran, I. Schoinas, R. Uhlig, B. Vembu, and J. Wiegert, "Intel virtualization technology for directed I/O," Intel technology journal, vol. 10, no. 3, 2006.

[8] G. Alendal, C. Kison, and modg, "got HW crypto? on the (in) security of a Self-Encrypting Drive series," techreport (2015), https://eprint.iacr. org/2015/1002.pdf.

[9] AMD.com, "AMD64 architecture programmer's manual volume 2: System programming," Dec. 2017. http://support.amd.com/TechDocs/ 24593.pdf.

[10] ArsTechnica, "New and improved CryptXXX ransomware rakes in $\$ 45,000$ in 3 weeks," news article (June 27, 2016). https://arstechnica.com/information-technology/2016/06/new-andimproved-cryptxxx-ransomware-rakes-in-45000-in-3-weeks/.

[11] J. V. Bulck, M. Minkin, O. Weisse, D. Genkin, B. Kasikci, F. Piessens, M. Silberstein, T. F. Wenisch, Y. Yarom, and R. Strackx, "Foreshadow: Extracting the keys to the intel SGX kingdom with transient out-of-order execution," in USENIX Security Symposium. USENIX Association, 2018, pp. 991-1008.

[12] K. R. B. Butler, S. McLaughlin, and P. D. McDaniel, "Rootkit-resistant disks," in ACM CCS'08, Alexandria, Virginia, USA, 2008.
[13] CardLogix.com, "Types of smart card," technical article (December, 2018). http://www.smartcardbasics.com/smart-card-types.html.

[14] A. Continella, A. Guagnelli, G. Zingaro, G. De Pasquale, A. Barenghi, S. Zanero, and F. Maggi, "ShieldFS: A self-healing, ransomware-aware filesystem," in ACSAC'16, Los Angeles, CA, USA, 2016.

[15] Drive Trust Alliance, "DTA sedutil self encrypting drive software," https://github.com/Drive-Trust-Alliance/sedutil.

[16] M. Ermolov and M. Goryachy, "How to hack a turned-off computer, or running unsigned code in Intel management engine," Blackhat Europe 2017.

[17] F-Secure, "The state of cyber security 2017," technical report (Feb. 16, 2017). https://www.f-secure.com/documents/996508/1030743/ cyber-security-report- 2017.

[18] S. Feaster, "FAT32-implementation," https://github.com/ ShamariFeaster/FAT32-Implementation.

[19] W. Garbe, "Computer attack erases Ohio police data," news article (May, 2018). https://www.officer.com/command-hq/technology/ computers-software/data-security/news/21005260/computer-attackerases-riverside-ohio-police-data.

[20] J. Götzfried, M. Eckert, S. Schinzel, and T. Müller, "Cache attacks on intel SGX," in European Workshop on Systems Security (EuroSec'17), Belgrade, Serbia, 2017.

[21] HealthcareITNews, "Ransomware: See the 14 hospitals attacked so far in 2016," news article (Oct. 5, 2016)

[22] HTBridge.com, "RansomWeb: emerging website threat that may outshine DDoS, data theft and defacements?" news article (Jan. 28, 2015). https://www.htbridge.com/blog/ransomweb_emerging_website_ threat.html.

[23] D. Y. Huang, D. McCoy, M. M. Aliapoulios, V. G. Li, L. Invernizzi, E. Bursztein, K. McRoberts, J. Levin, K. Levchenko, and A. C. Snoeren, "Tracking ransomware end-to-end," in IEEE SP'18.

[24] J. Huang, J. Xu, X. Xing, P. Liu, and M. K. Qureshi, "FlashGuard: Leveraging intrinsic flash properties to defend against encryption ransomware," in ACM CCS'17, Dallas, TX, USA, 2017.

[25] IEEE.org, "1667-2015 - IEEE standard for discovery, authentication, and authorization in host attachments of storage devices," https:// standards.ieee.org/findstds/standard/1667-2015.html.

[26] Intel.com, "Intel TXT software development guide, measured launched environment developer's guide," 2017, https://www.intel.com/ content/dam/www/public/us/en/documents/guides/intel-txt-softwaredevelopment-guide.pdf.

[27] D.-Y. Kao, "Rational choice observation of malware authors in taiwan," in Pacific-Asia Workshop on Intelligence and Security Informatics. Springer, 2014, pp. 1-7.

[28] B. Kauer, "OSLO: Improving the security of trusted computing," in USENIX Security Symposium, Boston, MA, USA, Aug. 2007.

[29] A. Kharaz, S. Arshad, C. Mulliner, W. Robertson, and E. Kirda, "UNVEIL: A large-scale, automated approach to detecting ransomware," in USENIX Security Symposium, Austin, TX, 2016.

[30] A. Kharraz and E. Kirda, "Redemption: Real-time protection against ransomware at end-hosts," in RAID'17, Atlanta, GA, USA, 2017.

[31] A. Kharraz, W. Robertson, D. Balzarotti, L. Bilge, and E. Kirda, "Cutting the Gordian knot: A look under the hood of ransomware attacks," in Detection of Intrusions and Malware, and Vulnerability Assessment (DIMVA), Milan, Italy, 2015.

[32] E. Kolodenker, W. Koch, G. Stringhini, and M. Egele, "PayBreak: Defense against cryptographic ransomware," in ACM Asia Conference on Computer and Communications Security (ASIACCS'17), Abu Dhabi, UAE, 2017, pp. 599-611.

[33] R. Krahn, B. Trach, A. Vahldiek-Oberwagner, T. Knauth, P. Bhatotia, and C. Fetzer, "Pesos: policy enhanced secure object store," in $E u$ roSys'18, Porto, Portugal, April 23-26, 2018, pp. 25:1-25:17.

[34] K. Kursawe, D. Schellekens, and B. Preneel, "Analyzing trusted platform communication," in ECRYPT Workshop, CRASH - CRyptographic Advances in Secure Hardware, Leuven, Belgium, Sep. 2005.

[35] A. Laszka, S. Farhang, and J. Grossklags, "On the economics of ransomware," CoRR, vol. abs/1707.06247, 2017. [Online]. Available: http://arxiv.org/abs/1707.06247 
[36] S. Lee, M.-W. Shih, P. Gera, T. Kim, H. Kim, and M. Peinado, "Inferring fine-grained control flow inside SGX enclaves with branch shadowing," in USENIX Security'17, Vancouver, BC, 2017, pp. 557-574.

[37] locmetrics.com, "Locmetrics," technical article (October, 2007). http: //www.locmetrics.com/index.html.

[38] LogRhythm Labs, "A technical analysis of WannaCry ransomware," technical report (May 16, 2017). https://logrhythm.com/blog/atechnical-analysis-of-wannacry-ransomware/.

[39] T. Longstaff, "Information about the PC CYBORG (AIDS) trojan horse," US DOE Computer Incident Advisory Capability (CIAC) information bulletin (Dec. 19, 1989). Wayback link: https://web.archive. org/web/20060610040400/http://ciac.org/ciac/bulletins/a-10.shtml.

[40] T. Mandt, M. Solnik, and D. Wang, "Demystifying the secure enclave processor," Azimuth Security and OffCell Research, Tech. Rep., 2016, black Hat Las Vegas.

[41] McAfee Labs Threat Advisory, "Ransomware-SAMAS," technical article (Mar. 17, 2017). https://kc.mcafee.com/resources/sites/MCAFEE/ content/live/PRODUCT_DOCUMENTATION/26000/PD26873/en_US/ McAfee_Labs_Threat_Advisory-Ransomware-SAMAS_v3.pdf.

[42] J. M. McCune, B. J. Parno, A. Perrig, M. K. Reiter, and H. Isozaki, "Flicker: An execution infrastructure for tcb minimization," in European Conference on Computer Systems (EuroSys'08), Glasgow, Scotland UK, 2008, pp. 315-328.

[43] C. Meijer and B. van Gastel, "Self-encrypting deception: weaknesses in the encryption of solid state drives (SSDs)," Radboud University and Open University of the Netherlands, Tech. Rep., 2018, https://www.ru. nl/publish/pages/909282/draft-paper.pdf.

[44] Microsoft, "Enable controlled folder access," online documentation (Aug. 25, 2017). https://docs.microsoft.com/en-us/windows/threatprotection/windows-defender-exploit-guard/enable-controlled-foldersexploit-guard.

[45] Microsoft.com, "Windows 10 on arm," technical article (December, 2018). https://docs.microsoft.com/en-us/windows/arm/.

[46] S. Mofrad, F. Zhang, S. Lu, and W. Shi, "A comparison study of Intel SGX and AMD memory encryption technology," in 7th International Workshop on Hardware and Architectural Support for Security and Privacy, ser. HASP'18, Los Angeles, California, 2018, pp. 9:1-9:8.

[47] T. Müller, T. Latzo, and F. C. Freiling, "Self-encrypting disks pose self-decrypting risks," in ACSAC'11, Orlando, FL, USA, 2011.

[48] T. Müller, H. Spath, R. Mäckl, and F. C. Freiling, "STARK tamperproof authentication to resist keylogging," in Financial Cryptography and Data Security (FC'13), Okinawa, Japan, Apr. 2013.

[49] M. Nemec, M. Sys, P. Svenda, D. Klinec, and V. Matyas, "The return of Coppersmith's attack: Practical factorization of widely used RSA moduli," in ACM CCS'17, Dallas, TX, USA, 2017.

[50] NetworkWorld.com, "The latest ransomware threat: Doxware," news article (Feb. 27, 2017). https://www.networkworld.com/article/3174678/ security/the-latest-ransomware-threat-doxware.html.

[51] Nuvoton.com, "Numicro m2351 series - a trustzone empowered microcontroller series focusing on iot security," product website (December, 2018). https://m2351.nuvoton.com/secure-microcontroller-platform/.

[52] PCWorld, "Alleged ransomware gang investigated by Moscow police," news article (Aug. 31, 2010). http://www.pcworld.com/article/204577/ article.html.

[53] r0m30, "msed - manage self encrypting drives," https://github.com/NPHardass/msed/tree/master/msed.

[54] Sakaki, "Sakaki's EFI install guide/disabling the intel management engine," wiki post (April 4, 2018). https://wiki.gentoo.org/wiki/Sakaki\% 27s_EFI_Install_Guide/Disabling_the_Intel_Management_Engine.

[55] N. Scaife, H. Carter, P. Traynor, and K. R. B. Butler, "Cryptolock (and drop it): Stopping ransomware attacks on user data," in International Conference on Distributed Computing Systems (ICDCS'16), Nara, Japan, Jun. 2016, pp. 303-312.

[56] Seagate, "DriveTrust technology: A technical overview," http://www seagate.com/docs/pdf/whitepaper/TP564_DriveTrust_Oct06.pdf.

[57] SecurityWeek, "LeChiffre ransomware hits Indian banks, pharma company," news article (Jan. 26, 2016). http://www.securityweek.com/ lechiffre-ransomware-hits-indian-banks-pharma-company.

[58] E. R. Sparks, "A security assessment of trusted platform modules,"
Dartmouth College, Tech. Rep., 2007, http://www.cs.dartmouth.edu/ reports/TR2007-597.pdf.

[59] W. Stallings, "Format-preserving encryption: Overview and NIST specification," Cryptologia, vol. 41, no. 2, pp. 137-152, 2017.

[60] J. D. Strunk, G. R. Goodson, M. L. Scheinholtz, C. A. N. Soules, and G. R. Ganger, "Self-securing storage: protecting data in compromised systems," in Foundations of Intrusion Tolerant Systems, 2003 [Organically Assured and Survivable Information Systems], Los Alamitos, CA, USA, 2003, pp. 195-209.

[61] R. Sturmer, "A lightweight implementation of the FAT32 filesystem for embedded systems," https://github.com/ryansturmer/thinfat32.

[62] ATA Security feature Set Clarifications, T13 Technical Committee, may 24, 2006.

[63] C. Tarnovsky, "Security failures in secure devices," Black Hat DC (Feb. 2008). https://www.blackhat.com/presentations/bh-dc-08/ Tarnovsky/Presentation/bh-dc-08-tarnovsky.pdf.

[64] The Atlantic, "The computer virus that haunted early AIDS researchers," news article (May 10, 2016). https://www.theatlantic.com/ technology/archive/2016/05/the-computer-virus-that-haunted-earlyaids-researchers $/ 481965 /$.

[65] The T13 Technical Committee, "Information technology - ATA command set - 4 (ACS-4)," http://www.t13.org/Documents/Uploaded Documents/docs2016/di529r14-ATAATAPI_Command_Set_-_4.pdf.

[66] TheRegister.co.uk, "South Korean hosting co. pays $\$ 1 \mathrm{~m}$ ransom to end eight-day outage," news article (June 20, 2017). https://www.theregister. co.uk/2017/06/20/south_korean_webhost_nayana_pays_ransom/.

[67] Trusted Computing Group, "TCG storage architecture core specification," https://trustedcomputinggroup.org/wp-content/uploads/TCG_ Storage_Architecture_Core_Spec_v2.01_r1.00.pdf.

[68] — "Trusted computing group and NVM express joint white paper: TCG storage, opal, and nvme," technical article (August, 2015). https://trustedcomputinggroup.org/wp-content/uploads/TCGandNVMe_ Joint_White_Paper-TCG_Storage_Opal_and_NVMe_FINAL.pdf

[69] TCG Storage Security Subsystem Class: Opal, Trusted Computing Group, August 2015.

[70] USB.org, "Enhanced host controller interface specification for universal serial bus," http://www.usb.org/developers/resources/.

[71] V. VENTURA and M. LEE, "Wiper malware: Attacking from inside," technical article (2018). https://talos-intelligencesite.s3.amazonaws.com/production/documentı_files/files/000/033/904/ original/Talos_WiperWhitepaper.v3.pdf.

[72] A. Winning, "First look at nordic's "cellular made easy" nrf91 low-power solution," news article (January, 2018). http://www.eenewsembedded.com/news/first-look-nordics-cellularmade-easy-nrf91-low-power-solution.

[73] J. Winter and K. Dietrich, "A hijacker's guide to communication interfaces of the trusted platform module," Computers and Mathematics with Applications, vol. 65, no. 5, pp. 748-761, Mar. 2013.

[74] Wired, "How an accidental 'kill switch' slowed Friday's massive ransomware attack," news article (May 13, 2017). https://www.wired.com/2017/05/accidental-kill-switch-slowed-fridaysmassive-ransomware-attack/.

[75] R. Wojtczuk and J. Rutkowska, "Attacking Intel trusted execution technology," black Hat DC (Feb. 2009). http://www.blackhat. com/presentations/bh-dc-09/Wojtczuk_Rutkowska/BlackHat-DC-09Rutkowska-Attacking-Intel-TXT-slides.pdf.

[76] R. Wojtczuk, J. Rutkowska, and A. Tereshkin, "Another way to circumvent Intel trusted execution technology," Invisible Things Lab, Tech. Rep., 2009, http://invisiblethingslab.com/resources/misc09/Another\% 20TXT\%20Attack.pdf.

[77] A. Woodward, "Bitlocker - the end of digital forensics?" in Australian Digital Forensics Conference, Perth, Western Australia, 2006, p. 38

[78] A. Young and M. Yung, "Cryptovirology: Extortion-based security threats and countermeasures," in IEEE Symposium on Security and Privacy, Oakland, CA, USA, May 1996.

[79] — "Cryptovirology: The birth, neglect, and explosion of ransomware," Communications of the ACM, vol. 60, no. 7, pp. 24-26, Jul. 2017.

[80] L. Ysboodt and M. D. Nil, "Embedded filesystems library," https:// sourceforge.net/projects/efsl/?source=typ_redirect. 